\title{
Theoretical investigation of the interfaces and mechanisms of induced spin polarization of 1D narrow zigzag graphene- and $h$-BN nanoribbons on SrO terminated LSMO(001) surface
}

\author{
Paul Avramov a,, , Alexandr A. Kuzubov ${ }^{\mathrm{b}, \mathrm{c}}$, Artem V. Kuklin ${ }^{\mathrm{a}, \mathrm{b}}$, Hyosun Lee ${ }^{\mathrm{a}}$, Evgenia A. \\ Kovaleva $^{\text {b,c }}$, Seiji Sakai ${ }^{\mathrm{d}}$, Shiro Entani ${ }^{\mathrm{d}}$, Hiroshi Naramoto ${ }^{\mathrm{d}}$ and Pavel B. Sorokin ${ }^{\mathrm{e}}$ \\ ${ }^{a}$ Department of Chemistry and Green-Nano Materials Research Center, Kyungpook National \\ University, 80 Daehak-ro, Buk-gu, Daegu, 41566, Republic of Korea \\ ${ }^{\mathrm{b}}$ Siberian Federal University, 79 Svobodniy pr., Krasnoyarsk 660041, Russia \\ ${ }^{c}$ L.V. Kirensky Institute of Physics SB RAS, 50/38 Akademgorodok, Krasnoyarsk 660036, \\ Russia \\ ${ }^{d}$ National Institutes for Quantum and Radiological Science and Technology QST, Tokai, Naka, \\ Ibaraki 319-1195, Japan \\ ${ }^{e}$ National University of Science and Technology MISiS, Moscow, 119049, Russia
}

The structure of the interfaces and the mechanisms of induced spin polarization of 1D infinite and finite narrow graphene- and $h$-BN zigzag nanoribbons placed on SrO-terminated $\mathrm{La}_{1}$ ${ }_{x} \mathrm{Sr}_{x} \mathrm{MnO}_{3}$ (LSMO) (001) surface were studied using Density Functional (DFT) electronic structure calculations. It was found that the $\pi$-conjugated nanofragments are bounded with LSMO(001) surface by weak disperse interactions. The types of coordination of the fragments, the strength of bonding and the rate of spin polarization depend upon the nature of the fragments. Infinite and finite graphene narrow zigzag nanoribbons are characterized by the lift of the spin degeneracy and strong spin-polarization caused by interface-induced structural asymmetry and oxygen-mediated indirect exchange interactions with Mn ions of LSMO support. Spin polarization changes the semiconducting nature of infinite graphene nanoribbons to half-metallic state with visible spin-up density of states at the Fermi level. The $h$-BN nanoribbon binding energy is weaker than graphene nanoribbon ones with noticeably shorter interlayer distance. The asymmetry effect and indirect exchange interactions cause spin polarization of $h$-BN nanoribbon as well with formation of embedded states inside the band gap. The results show a possibility to use one-atom thick nanofragments to design LSMO-based heterostructures for spintronic nanodevices with $h$-BN as an inert spacer to develop different potential barriers.

Keywords: LSMO thin film, pentacene, spin states, induced spin polarization, graphene and $h$ $\mathrm{BN}$ nanoribbons, heterostructures, $\mathrm{DFT}+\mathrm{U}$ calculations.

*Author to whom correspondence should be addressed.

E-mail: paul@iph.krasn.ru 


\section{Introduction}

The progress in developing of graphene-based spintronic nanodevices is related with weak spin-orbital interactions of carbon atoms, which enable one to utilize extremely high mobility of electrons together with long spin transport length. ${ }^{1-3}$ The effectiveness of spin injection in carbon nanostructures by ferromagnetic supports (e.g. Ni(111)) has already been discovered experimentally and interpreted theoretically. ${ }^{4,5}$ In particular, high magnetoresistance has been detected in $\pi$-conjugated organic media on ferromagnetic supports. ${ }^{6-10}$ Isolating $h$-BN fragments located between graphene and ferromagnetic metallic supports have been used for chemical passivation of the surfaces and creation of tunnel contacts to promote graphene spin injection. ${ }^{11-13}$ It was demonstrated that in 2D nanoheterostructures the injection coherence length could achieve up to $350 \mu \mathrm{m}^{14,15}$ due to interface-related Buchkov-Rashba effect. ${ }^{16,17,18}$

Bychkov-Rashba effect is caused by the lift of spin degeneracy due to structure inverse asymmetry (SIA) ${ }^{18}$ in $2 \mathrm{D}$ electron gas $(2 \mathrm{DEG})$ at the interfaces. $2 \mathrm{DEG}$ is confined by the effective electric field oriented in [001] direction caused by SIA effect, which, in many cases, is much stronger than the bulk symmetrical features. ${ }^{19}$ For nonmagnetic surfaces, the Rashba field lifts the spin degeneracy of the surface states, ${ }^{19}$ whereas for magnetic ones, it leads to an asymmetric shift of the spin nondegenerate bands. ${ }^{20}$

Using both experimental and theoretical approaches it was found that indirect exchange interactions between transitional metal ions mediated by oxygen ions of the crystalline lattices play a critical role in formation of the main features in the electronic structure of complex manganites ${ }^{21,22}$ in accordance with RKKY mechanism. ${ }^{23-25}$ The photoemission and X-ray spectroscopy ${ }^{26,27}$ revealed antiferromagnetic bonding of the oxygen holes with spin configurations of $\mathrm{Mn}^{+3}$ ions in $\mathrm{La}_{1-\mathrm{x}} \mathrm{Sr}_{\mathrm{x}} \mathrm{MnO}_{3}$. The electron-hole excitation leads to charge transfer from $\mathrm{O} 2 p$ sub-band to $\mathrm{Mn}^{3+}$ ions with the formation of $\mathrm{Mn}^{4+}$ configuration through the double exchange mechanism. ${ }^{28}$

Perovskite manganites are well known as an advantage class of functional magnetic materials with a rich variety of magnetic and electric properties. A half-metallic manganite class $\mathrm{La}_{1-x} \mathrm{Sr}_{x} \mathrm{MnO}_{3}$ (LSMO) is a promising material for spintronic applications due to $100 \%$ spin polarization, efficient spin injection, colossal magnetoresistance, low-density of charge carriers $\left(10^{21}-10^{22} \mathrm{~cm}^{-3}\right)$, the highest Curie temperature $\left(\mathrm{T}_{\mathrm{c}}=370 \mathrm{~K}\right)$ and partial transparency. ${ }^{29-33}$ Due to these properties, LSMO is a good choice to design tunnel magnetic contacts. ${ }^{34}$ Using spinpolarized currents through LSMO-graphene interfaces a number of LSMO-based high-efficiency organic LED ${ }^{35}$ and spin-valve nanodevices ${ }^{36-38}$ have been developed. High sensitivity of organicbased heterostructures was demonstrated for a photodetector driven by ferroelectrics. ${ }^{39}$ The proximity effect of spin-polarization of $2 \mathrm{D}$ graphene at $\mathrm{La}_{0.7} \mathrm{Sr}_{0.3} \mathrm{MnO}_{3}$ was studied by a combined experimental and theoretical approach ${ }^{40}$ and the direct evidence of sizable spin polarization at the Fermi level of 2D graphene parallel to the spin polarization of LSMO was obtained.

Large magnetoresistance effects coupled with large output signals were detected in a nanodevice of multiwall carbon nanotube that spans a $1.5 \mathrm{~mm}$ gap between spin-polarized half- 
metallic $\mathrm{La}_{0.7} \mathrm{Sr}_{0.3} \mathrm{MnO}_{3}$ electrodes. ${ }^{41}$ The device demonstrated long spin lifetime and high Fermi velocity in the nanotube, the high spin polarization of the manganite electrodes and the resistance of the interfacial barrier for spin injection. The experimental results were supported by density functional theory calculations.

Other examples of the key $\pi$-conjugated media to promote spin injection are pentacene molecule $\mathrm{C}_{22} \mathrm{H}_{14}$, which is, in fact, a short finite narrow graphene zigzag nanoribbon of five hexagonal ring length and one hexagonal ring width and infinite zigzag graphene nanoribbons (ZGNRs) of different width. It was demonstrated that vertical pentacene-based spintronic devices with $\mathrm{La}_{0.7} \mathrm{Sr}_{0.3} \mathrm{MnO}_{3}$ and $\mathrm{Co}$ electrodes retain magnetoresistive effects up to room temperature. ${ }^{42}$ Recently, spin-dependent molecular symmetry associated with $p-d$ hybridization between pentacene and cobalt nanoscale islands deposited on $\mathrm{Cu}(111)$ were studied by SP-STM technique $^{43}$ which allows one to predict and control induced spin polarization of $\pi$-conjugated molecules on magnetic substrates. ZGNRs show antiferromagnetic semiconducting nature ${ }^{44}$ with ferromagnetic spin ordering at each edge. ${ }^{45}$ It is worth noting that ZGNRs demonstrate singlechannel room temperature ballistic conductivity on a length scale greater than theoretically predicted for perfect graphene and equal to ten micrometers. ${ }^{46,47}$ Thus, it offers a possibility to create novel LSMO-based heterostructures with induced half-metallic ferromagnetic nature for promising spintronics applications.

In the present paper, the atomic and electronic structure and spin states of novel lowsymmetry low-dimensional heterostructures constituted by finite and infinite graphene and $h$-BN zigzag nanoribbons deposited on SrO-terminated LSMO(001) surface are studied using state-ofthe-art Density Functional technique and interpreted in terms of indirect exchange interactions between the fragments. The types of coordination of the fragments, the nature and strength of the bonding and the fragment rates of induced spin polarization are of special interest of the study.

\section{Computational methods and models}

To study the atomic and electronic structure of LSMO-based low-dimensional heterostructures, density functional theory (DFT) and general gradient approximation (GGA) Perdew, Burke, Ernzerhof (PBE) potential ${ }^{48}$ within the framework of periodic boundary conditions (PBC) were used as implemented in the VASP code ${ }^{49,50}$ with taking into account Hubbard correlations $(\mathrm{GGA}+\mathrm{U})^{51,52}$ and empirical D3 Grimme corrections of weak dispersion interactions. ${ }^{53}$ For all calculations a plane-wave basis set within the projector augmented wave (PAW) method ${ }^{54,55}$ was used. The GGA+U parameters $U=2$ and $J=0.7 \mathrm{eV}$ were adopted from earlier calculations of LSMO ${ }^{56-58}$ For all calculations, the cutoff energy ( $\left.E_{\text {cutoff }}\right)$ was set to 450 $\mathrm{eV}$. The geometry of the heterostructures was fully optimized (the coordinates of the atoms in the supercells and all translational vectors) until the residual forces became less than $0.01 \mathrm{eV} / \AA$. A vacuum region of $17 \AA$ was set to avoid artificial interaction between neighboring supercell images. For pristine LSMO, the $\Gamma$-centered Monkhorst-Pack ${ }^{59}(12 \times 12 \times 12 k$-point set $)$ scheme was used. For test calculations, $(18 \times 18 \times 18)$ sampling was employed and it was found that the energy difference between two types of sampling is less $0.01 \mathrm{eV} / \mathrm{unit}$ cell. 
For $\mathrm{La}_{0.67} \mathrm{Sr}_{0.33} \mathrm{MnO}_{3}$ with pseudocubic perovskite-type unit cell, the $a$ translation vector was found to be equal to $3.886 \AA$ which is in a good agreement with experimental data ( $a=3.876$ $\AA^{60}$ and $\left.a=3.87 \AA^{61}\right)$ and previous theoretical calculations $(a=3.89 \AA) .{ }^{56}$ The GGA+U D3 PBC PAW test calculations of 2D graphene and $h-\mathrm{BN}$ revealed C-C and B-N distances equal to 1.425 and $1.470 \AA$, respectively, which perfectly coincides with well-known experimental data of $1.423 \AA$ and $1.464 \AA$, respectively, which can be easily found elsewhere. Three infinite and one finite one-dimensional narrow zigzag graphene nanoribbons were used to design the composites, namely: pentacene molecule $\mathrm{C}_{22} \mathrm{H}_{14}$, which consists of five linearly-fused benzene rings (in fact the molecule is a short finite fragment of narrowest zigzag graphene nanoribbon); narrow zigzag graphene nanoribbon of one carbon hexagon width (standard notation 2-ZGNR) and 3 carbon hexagon length of the unit cell $\left(\mathrm{C}_{12} \mathrm{H}_{6}\right.$ supercell, 18 carbon and hydrogen atoms in total); zigzag graphene nanoribbon of three carbon hexagon width (standard notation 4-ZGNR) and 3 carbon hexagon length in the unit cell $\left(\mathrm{C}_{24} \mathrm{H}_{6}\right.$ supercell, 30 carbon and hydrogen atoms in total). Only one low-dimensional $h$-BN fragment, namely zigzag $h$-BN nanoribbon of three $\mathrm{B}_{3} \mathrm{~N}_{3}$ hexagon width (4-ZBNNR) and $3 \mathrm{~B}_{3} \mathrm{~N}_{3}$ hexagon length $\left(\mathrm{B}_{12} \mathrm{~N}_{12} \mathrm{H}_{6}\right.$ supercell, 30 boron, nitrogen and hydrogen atoms in total) was used to simulate LSMO(001)-based heterostructure with $1 \mathrm{D} h$-BN zigzag nanoribbon. 47 different initial mutual configurations of the zigzag nanoribbons with LSMO(001) surface were probed for conformational scan with 13 stable and metastable interface structures finally located by full optimization procedure (all located configurations will be discussed in details below). To perform full optimization, the initial distance along z-direction for all configurations was chosen to be $3.5 \AA$. For the sake of comparison free-standing LSMO(001), pentacene molecule, 2-ZGNR, 4-ZGNR and 4-ZBNNR fragments were calculated as well.

Special attention was paid to achieve high-accuracy results in ab initio DFT calculations. Two types of plates of LSMO(001) thin film with different thickness with 3 and 6 pseudocubic unit cells thickness were tested and it was found that the surface energy difference between them is less than $0.01 \mathrm{eV} / \AA^{2}$. The charge distributions of Sr and $\mathrm{O}$ surface atoms for 3 and 6 unit cell thickness of thin film were compared by using Bader code. ${ }^{62-64}$ It was found that strontium and oxygen ions bear $1.54 e$ and $-1.37 e$ charges, respectively, for 3 unit cell and $1.54 e$ and $-1.27 e$ charges, respectively, for 6 unit cell thicknesses. One can see some small difference for the atomic charge of $\mathrm{O}$ ions, while the charge state of $\mathrm{Sr}$ ions remains intact. Since the difference in charge states of surface ions is small, for practical reasons for electronic structure calculations a slab of 3 unit cell thickness was adopted. The dependence of the total energies upon the vacuum distances was tested up to $24 \AA$ along $c$ vector and it was found that no energy dependence upon the vacuum interval is detected for the length longer $15 \AA$. It is necessary to note that the effective distances for van-ver-Waals $(\mathrm{vdW})$ forces interactions are equal to 3-6 $\AA .{ }^{65}$

The LSMO(001) surface can be terminated by either $\mathrm{MnO}_{2^{-}}{ }^{66-68}$, SrO- or mixed $\mathrm{MnO}-$ $\mathrm{SrO}^{69}$ terminal layers with dominant $\mathrm{Sr}$ occupation. The SrO-terminated LSMO thin film was developed as a limiting case of $\operatorname{LSMO}(001)$ surface following a number of experimental and theoretical publications. ${ }^{70-72}$ The LSMO(001) plate consists of three $\mathrm{MnO}$, two $\mathrm{LaO}$ and one top 
interface SrO layer (Figure 1a). The $\mathrm{Mn}$ ions are six-coordinated and $\mathrm{Sr}$ and $\mathrm{La}$ ions are fourcoordinated. La and Sr ions form separate layers and occupy the centers of the cubes formed by Mn-O units.

Two types of LSMO plate slabs were used for electronic structure calculations of the composites (Figure 1b): $\mathrm{La}_{20} \mathrm{Sr}_{10} \mathrm{Mn}_{30} \mathrm{O}_{90}$ (150 atoms) was used for pentacene/LSMO(001) heterostructure and consisted of $5 \times 2 \times 1$ structural units of bulk LSMO along $a$ (23.316 $\AA$ ), $b$ (7.772 $\AA$ ) and $c(30.00 \AA)$ directions, respectively; $\mathrm{La}_{32} \mathrm{Sr}_{16} \mathrm{Mn}_{48} \mathrm{O}_{144}$ (240 atoms) was used for 2ZGNR/LSMO(001), 4-ZGNR/LSMO(001) and 4-ZBNNR/LSMO(001), with $8 \times 2 \times 1$ structural units along $a(31.09 \AA), b(7.772 \AA)$ and $c(30.00 \AA)$ translation vectors respectively. The lateral distances between the images of 2-ZGNR and 4-ZGNR/4-ZBNNR in $a$ direction were equal to 26.12 and $21.86 \AA$, respectively. For pentacene molecule, the $a$ and $b$ intervals were equal 5.22 and $2.77 \AA$, respectively. Due to computational limitations all carbon and $h$-BN nanoribbons were oriented only along $b$ direction.

a)

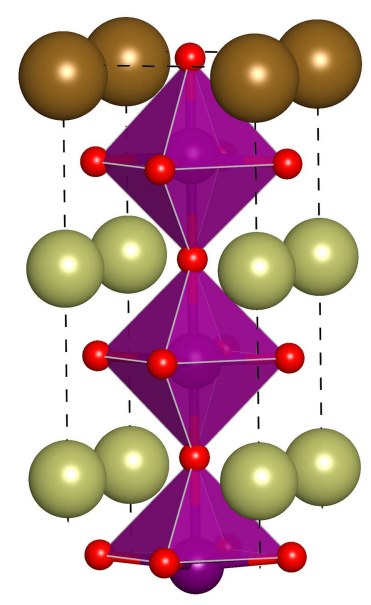

b)
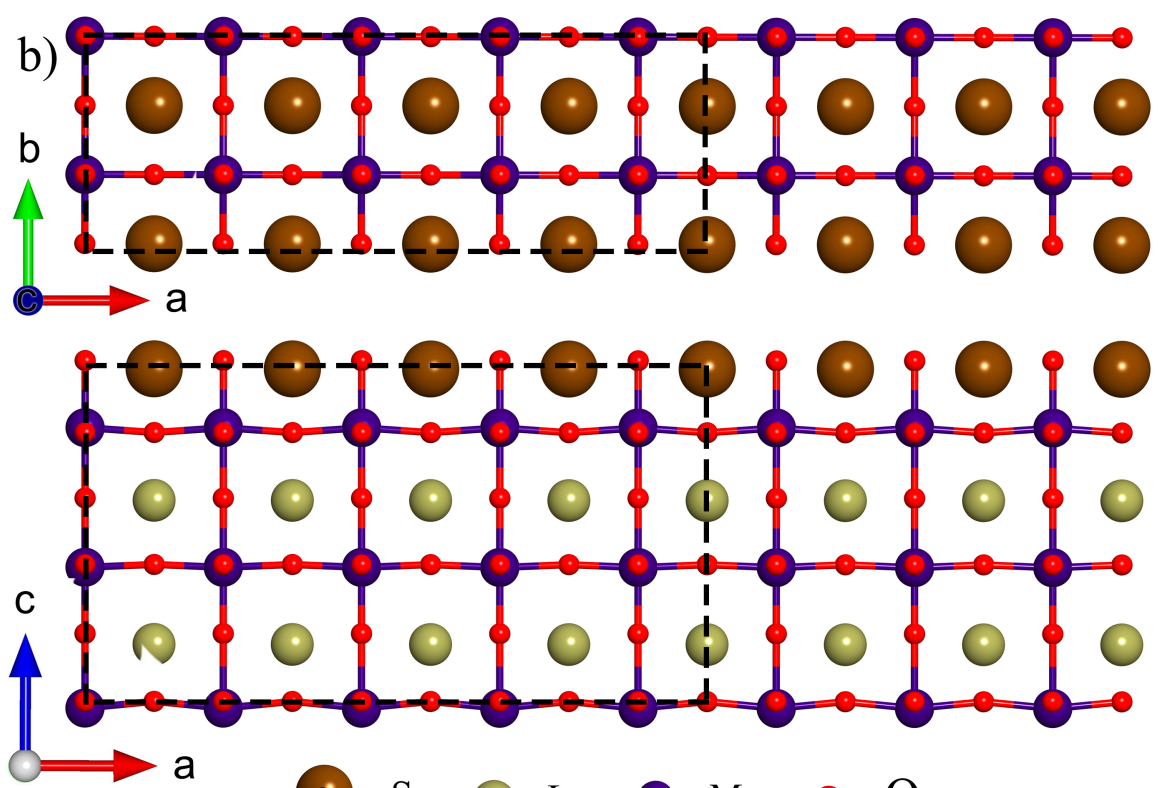

(3) - $\mathrm{Sr} \odot-\mathrm{La} \odot-\mathrm{Mn} \quad \odot-\mathrm{O}$

Figure 1. a) The atomic structure of bulk LSMO unit cell with one $\mathrm{SrO}$, two $\mathrm{LaO}$ and three $\mathrm{MnO}_{6}$ layers. b) Atomic structure of LSMO thin film used as a support for low-dimensional graphene- and $h$-BN fragments. The top and side views of LSMO(001) thin film with outlined supercells are presented at top-right and bottom-right of the figure. The $5 \times 2 \times 1$ supercell boundaries for pentacene/LSMO(001) are marked by dashed lines (150 atoms in the slab). The entire $8 \times 2 \times 1$ structure (240 atoms) corresponds to LSMO slab for 2-ZGNR/LSMO(001), 4ZGNR/LSMO(001) and 4-ZBNNR/LSMO(001) heterostructures.

The binding energies of 2-ZGNR, 4-ZGNR, pentacene and 4-ZBNNR with LSMO(001) substrate were calculated using the equation 


$$
E_{B}=E_{T}-E_{\text {LSMO }}-E_{\text {frag }}(1)
$$

where $E_{T}$ is the total energy of the composites, $E_{L S M O}$ is the energy of pristine LSMO slab and $E_{\text {frag }}$ is the energy of freestanding low-dimension fragment, either 2-ZGNR, 4-ZGNR, pentacene molecule or 4-ZBNNR.

\section{Results and discussion}

The electronic structure calculations of all LSMO slabs reveal small rotations of $\mathrm{MnO}_{6}$ octahedrons (in order of $1^{\circ}$ ) along $c$ direction, which coincide well with previous experimenta $1^{73}$ and theoretical ${ }^{74}$ publications. Formation of $\mathrm{SrO}$ surface leads to a breakdown of perfect $\mathrm{SrO}$ plane of bulk LSMO with the value of corrugation of $0.230 \AA$ along $c$ direction between $\mathrm{Sr}$ and $O$ ions. The weak interactions between $\operatorname{LSMO}(001)$ surface from one side and pentacene/2ZGNR/4-ZGNR/4-ZBNNR fragments also lead to breakdown of perfect flat structures of pentacene, 4-ZGNR and 4-ZBNNR with $0.020 \AA, 0.082 \AA$ and $0.330 \AA$ corrugation values, respectively. It is necessary to note that the large value of corrugation for $h$-BN nanoribbon is caused by electrostatic attraction $(\mathrm{O}$ and $\mathrm{B})$ and repulsion $(\mathrm{O}$ and $\mathrm{N})$ of the ions of LSMO surface and $h$-BN fragment, respectively.

To define coordination types of pentacene molecule, 2-ZGNR and 4-ZGNR on SrOterminated surface, standard notations of coordination sites $\eta^{l}, \eta^{2}, \eta^{3}$ and $\eta^{6}$ are used throughout the text to denote the coordination of $\mathrm{Sr}$ surface ions to single carbon atom, the middle of $\mathrm{C}-\mathrm{C}$ bond, $\mathrm{C}_{3}$ triangle fragment and the center of $\mathrm{C}_{6}$ hexagons, respectively. For 4-ZBNNR the same notations are used with $\eta^{2}$ and $\eta^{6}$ correspond to the middle of B-N bond and center of $\mathrm{B}_{3} \mathrm{~N}_{3}$ hexagon. For $h$-BN, the $\eta^{l}$ and $\eta^{3}$ coordinations are special and reflect both $\mathrm{B}$ and $\mathrm{N}$ ( $\eta^{l}$ type) and $\mathrm{B}_{2} \mathrm{~N}$ and $\mathrm{BN}_{2}$ ( $\eta^{3}$ type) sites, respectively. It is necessary to note that 4-ZBNNR/LSMO(001) interfaces with $\eta^{l}$ and $\eta^{3}$ display all coordination types simultaneously and the notations do not reflect completely the way 4-ZBNNR is bound to LSMO(001) substrate. The combined types of configurations like $\eta^{l-2}$ or $\eta^{2-6}$ denote bonding of Sr ions to $\eta^{l}$ and $\eta^{2}$ or $\eta^{2}$ and $\eta^{6}$ sites, respectively. For complete description of the way the fragments bound to the substrate, specific illustrations are referred in the text.

Only four 2-ZGNR/LSMO(001) (Table 1, Figures 2a-2d) and three pentacene/LSMO(001) (Table 1, Figures 2e-2g) interface configurations are localized by electronic structure calculations. 

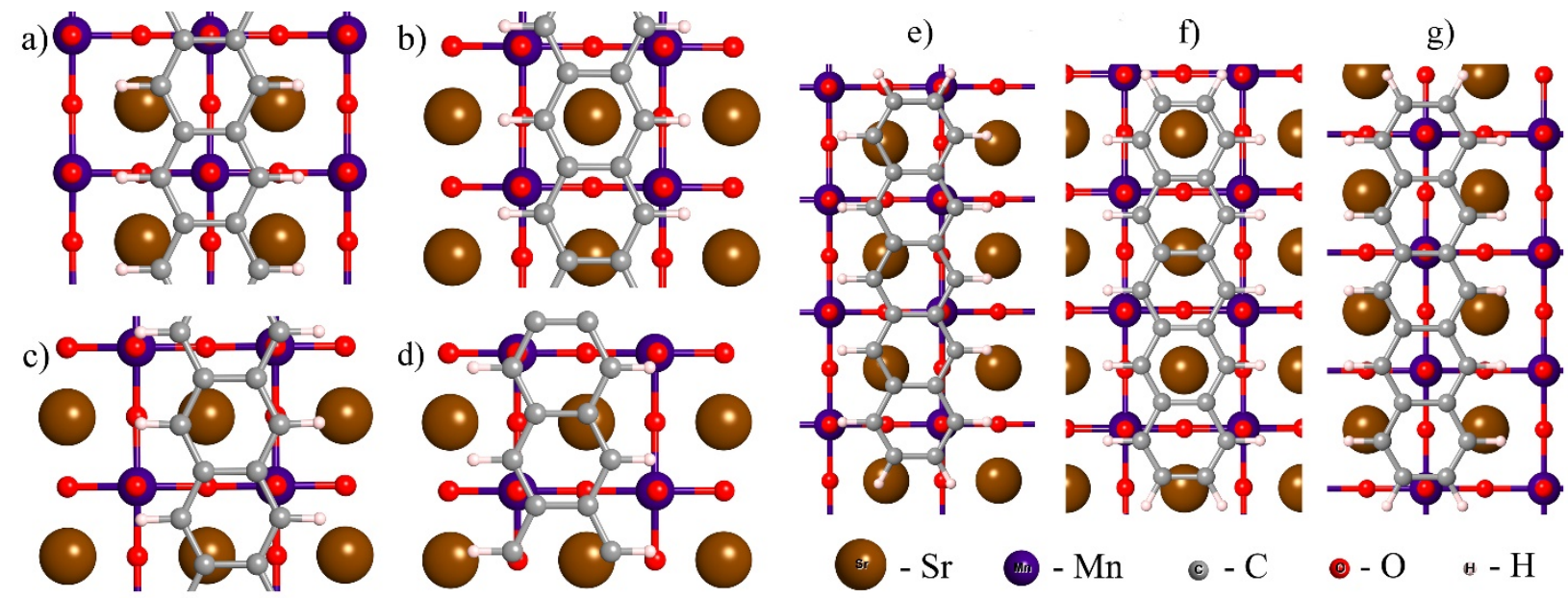

Figure 2. Four (a, b, c, d) and three (e, f, g) interface configurations of 2-ZGNR/LSMO(001) and pentacene/LSMO(001) heterostructures, respectively, located using electronic structure calculations. a) $\eta^{l}$ configuration with carbon atoms coordinated atop $\mathrm{Sr}$ ions; b) $\eta^{2-6}$ configuration with $\mathrm{Sr}$ ions coordinated to the middle of $\mathrm{C}-\mathrm{C}$ bonds and to the centers of carbon hexagons; c) $\eta^{2}$ and d) $\eta^{l-2}$ configurations with $\mathrm{Sr}$ ions coordinated to $\mathrm{C}-\mathrm{C}$ bonds and atop carbon atoms and C-C bonds, respectively. e) $\eta^{1-2}$ coordination of pentacene molecule through the bonding of Sr ions with carbon atoms and C-C bonds; f) $\eta^{2-6}$ coordination through C-C bonds and $\mathrm{C}_{6}$ hexagons and $\mathrm{g}$ ) $\eta^{I}$ configuration atop carbon atoms.

Table 1. Binding energies and interlayer distances of 2-ZGNR/LSMO $(001)$ and pentacene/LSMO $(001)$ heterostructures. The energetically favorable interface configurations $\left(\eta^{l}\right.$ type for both 2-ZGNR/LSMO(001) and pentacene/LSMO(001) heterostructures) are characterized by the negative binding energies with the biggest absolute values.

\begin{tabular}{|c|c|c|c|c|}
\hline \multirow{2}{*}{ Configuration } & \multicolumn{4}{|c|}{ 2-ZGNR/LSMO(001) } \\
\hline & $\eta^{l}$ & $\eta^{2-6}$ & $\eta^{2}$ & $\eta^{l-2}$ \\
\hline $\begin{array}{l}\text { Binding energy, eV } \\
\text { (Binding energy per } \\
\text { carbon atom, eV) }\end{array}$ & $\begin{array}{l}-0.905 \\
(-0.075)\end{array}$ & $\begin{array}{l}-0.525 \\
(-0.044)\end{array}$ & $\begin{array}{l}-0.753 \\
(-0.063) \\
\end{array}$ & $\begin{array}{l}-0.646 \\
(-0.054) \\
\end{array}$ \\
\hline $\begin{array}{c}\text { Average interlayer } \\
\text { distance, } \AA\end{array}$ & 3.192 & 3.227 & 3.203 & 3.217 \\
\hline \multirow{2}{*}{ Configuration } & \multicolumn{4}{|c|}{ Pentacene/LSMO(001) } \\
\hline & $\eta^{l-2}$ & & $\eta^{2-6}$ & $\eta^{l}$ \\
\hline $\begin{array}{l}\text { Binding energy, eV } \\
\text { (Binding energy per } \\
\text { carbon atom, eV) }\end{array}$ & $\begin{array}{r}-2.269 \\
(-0.103) \\
\end{array}$ & & $\begin{array}{l}-1.901 \\
(-0.086) \\
\end{array}$ & $\begin{array}{l}-2.355 \\
(-0.107) \\
\end{array}$ \\
\hline $\begin{array}{l}\text { Average interlayer } \\
\text { distance, } \AA\end{array}$ & 3.137 & & 3.154 & 3.099 \\
\hline
\end{tabular}


All located interface configurations of 2-ZGNR/LSMO(001) and pentacene/LSMO(001) heterostructures are presented in Table 1 . The energetically favorable interface configurations are characterized by the negative binding energies with the biggest absolute values. The binding energies per carbon atom and interlayer distances of 2-ZGNR/LSMO(001) and pentacene/LSMO(001) heterostructures reveal weak binding nature of LSMO support with both 2-ZGNR and pentacene. For 2-ZGNR/LSMO(001), the energetically preferable $\eta^{l}$ configuration is characterized by the binding energy of $-0.075 \mathrm{eV} /$ carbon atom and $3.192 \AA$ interlayer distance. The pentacene/LSMO(001) heterostructure also reveals $\eta^{l}$ configuration as the energetically preferable one. Pentacene is slightly stronger bounded with LSMO(001) substrate with bonding energy equal to $-0.107 \mathrm{eV} /$ carbon atom. The interlayer distance for pentacene $\eta^{l}$ configuration is the shortest one (3.099 $\AA$ ).

Six possible configurations of $4-Z G N R / L S M O(001)$ heterostructure are revealed by electronic structure calculations, namely: a) $\eta^{2}$; b) $\eta^{2-6}$; c) $\eta^{2-3}$; d) $\eta^{3}$; e) $\eta^{1-2-3-6}$ and f) $\eta^{l}$. The structures and parameters of all configurations are presented in Figure 3 and Table 2. Energetically preferable $\eta^{3}$ 4-ZGNR/LSMO(001) configuration with the lowest binding energy $(-0.105 \mathrm{eV} /$ carbon atom) is characterized by $3.068 \AA$ interlayer distance. The highest in energy $\eta^{l}$ configuration $(-0.026 \mathrm{eV} /$ carbon atom) has $3.141 \AA$ interlayer distance.

a)

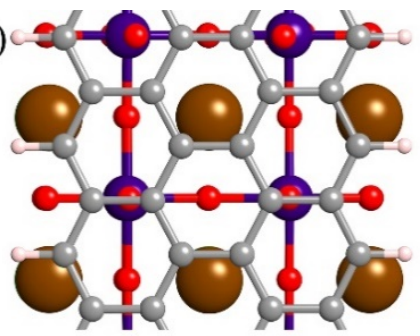

b)

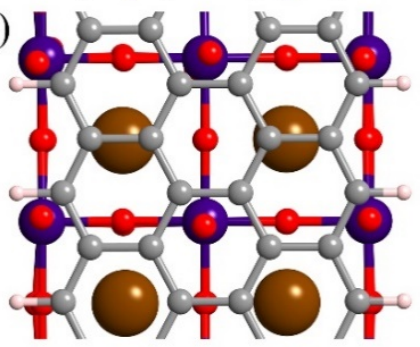

c)
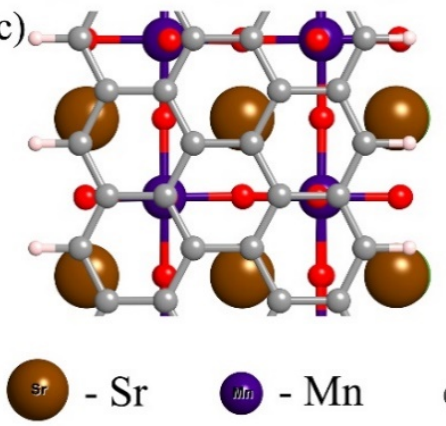

d)

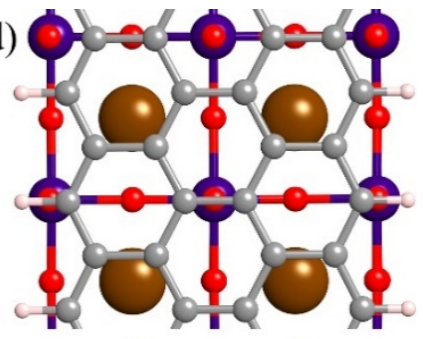

e)
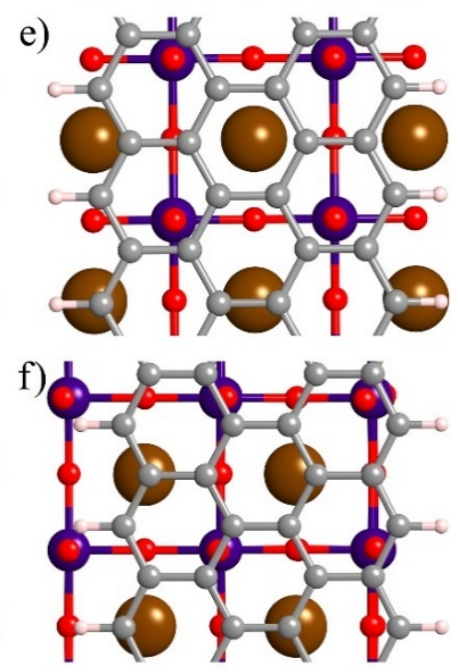

c $-\mathrm{C}$ - $-\mathrm{O}$ ㄱ- $\mathrm{H}$ 
Figure 3. Six located interfaces of 4-ZGNR/LSMO(001) heterostructure. a) $\eta^{2}$ configuration; b) $\eta^{2-6}$ configuration; c) $\eta^{2-3}$ configuration; d) $\eta^{3}$ configuration; e) $\eta^{1-2-3-6}$ configuration; f) $\eta^{l}$ configuration.

Table 2. Binding energies and average interlayer distances of 4-ZGNR/LSMO(001) heterostructures.

\begin{tabular}{ccccccc}
\hline \multirow{2}{*}{ Configuration } & $\eta^{2}$ & $\eta^{2-6}$ & $\eta^{2-3}$ & $\eta^{3}$ & $\eta^{1-2-3-6}$ & $\eta^{l}$ \\
\cline { 2 - 6 } & -0.935 & -0.745 & -1.163 & -2.524 & -2.064 & -0.623 \\
\hline $\begin{array}{c}\text { Binding energy, eV } \\
\begin{array}{c}\text { Binding energy per } \\
\text { carbon atom, eV) }\end{array}\end{array}$ & $(-0.039)$ & $(-0.031)$ & $(-0.048)$ & $(-0.105)$ & $(-0.086)$ & $(-0.026)$ \\
\hline $\begin{array}{c}\text { Average interlayer } \\
\text { distance, } \AA\end{array}$ & 3.123 & 3.132 & 3.119 & 3.068 & 3.096 & 3.141 \\
\hline
\end{tabular}

The binding energies of energetically favorite configurations of 2-ZGNR/LSMO(001), pentacene/LSMO(001) and 4-ZGNR/LSMO(001) heterostructures. $(-0.075--0.107 \mathrm{eV} /$ atom, Tables 1,2$)$ are close to the upper limit of the energies of van der Waals (vdW) bonds $(-0.043$ $-0.004 \mathrm{eV} /$ atom), ${ }^{65}$ which indicates negligible role of chemical binding (either covalent, complex or ionic) between LSMO and graphene nanofragments. The interfaces of all three carbon-based heterostructures are characterized by low-order coordination types $\eta^{l}, \eta^{l}$ and $\eta^{3}$ for pentacene/LSMO(001), 2-ZGNR/LSMO(001) and 4-ZGNR/LSMO(001), respectively. Very unusual coordination types of the fragments can be interpreted as clear manifestation of vdW bonding between the fragments.

Formation of the heterostructures leads to fundamental lowering of the symmetry of the electronic structure of the fragments at the interfaces. Combined with indirect electronic exchange with LSMO substrate, it lifts the spin degeneration of all 1D $\pi$-conjugated fragments. The total and partial density of states (TDOS and PDOS, respectively) and spatial distribution of spin density of pentacene/LSMO(001) and 2-ZGNR/LSMO(001) heterostructures are presented in Figures $4 \mathrm{a}$ and $4 \mathrm{~b}$, respectively. The shapes of TDOSes of both heterostructures are determined by LSMO(001) substrate. Freestanding pentacene molecule has closed-shell electronic structure with HOMO-LUMO gap equal to $0.9 \mathrm{eV}$. The result of the formation of the interface with LSMO can be easily followed by comparison of TDOS of free-standing pentacene (red curves on the bottom of Figure 4a) with PDOS of pentacene fragment in pentacene/LSMO(001) heterostructure (blue curves). One can easily detect significant $(0.9 \mathrm{eV})$ blue energy shift of the spin-down pentacene quasi-molecular electronic subsystem with almost intact spin-up quasi-molecular states. The indirect electronic exchange between pentacene and LSMO(001) converts diamagnetic pentacene molecule into $100 \%$ spin-polarized $\pi$-conjugated carbon nanofragment. In particular, the spin-up HOMO state demonstrates visible non-zero electronic density at the Fermi level. 


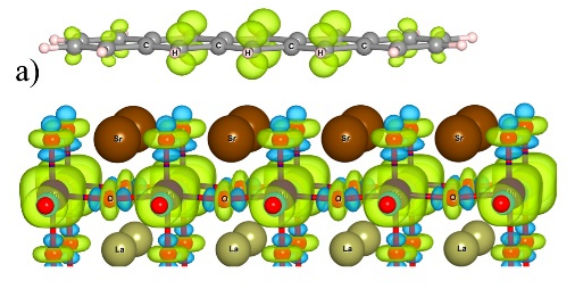

b)
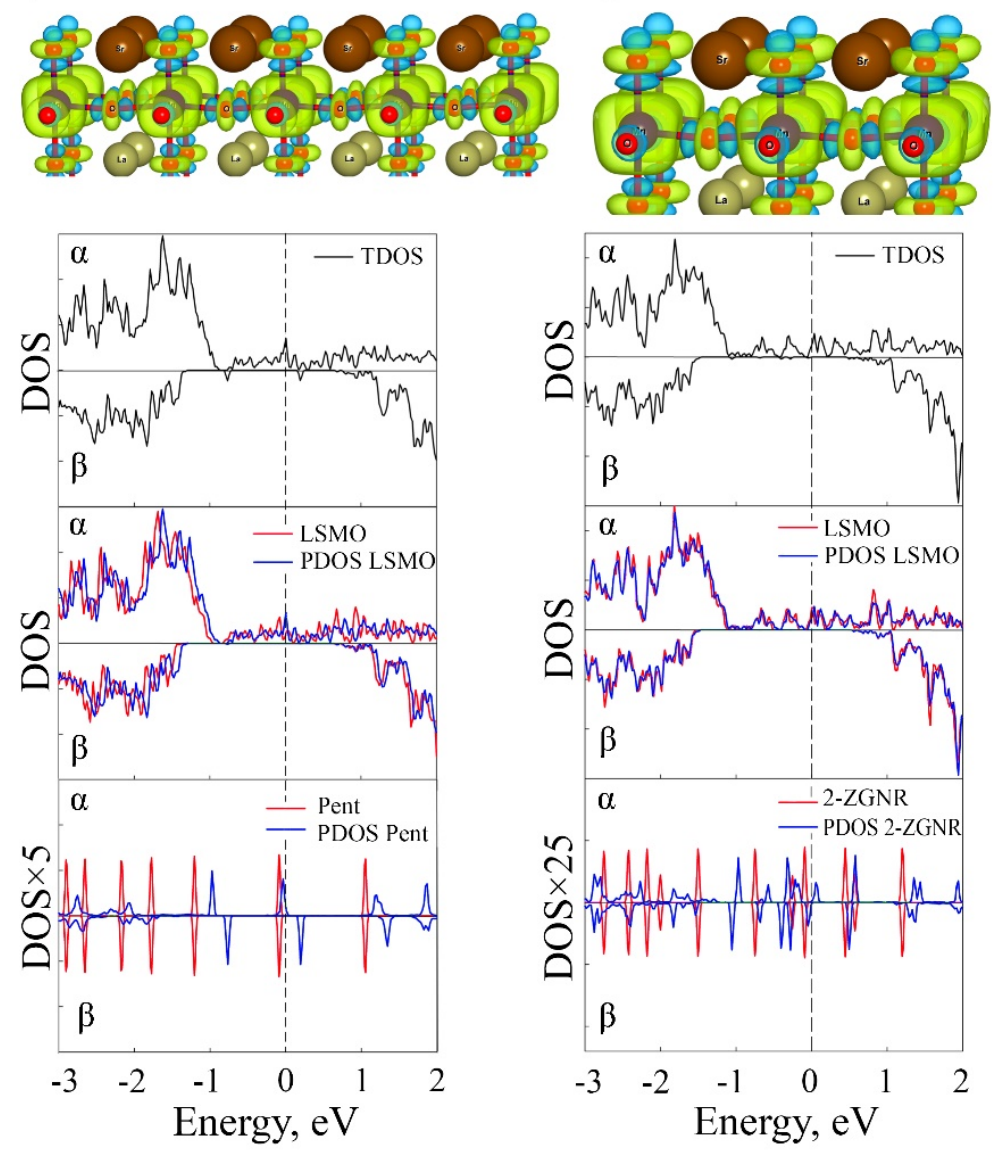

Figure 4. Spatial distribution of spin density (top of the Figure, left and right) and total and partial density of states (TDOS and PDOS, respectively) of (a) pentacene/LSMO(001) and (b) 2ZGNR/LSMO(001) heterostructures in energetically favorable $\eta^{l}$ configurations (Table 1). Density of states from top to bottom for both heterostructures: (top) TDOSes of pentacene/LSMO(001) (a) and 2-ZGNR/LSMO(001) (b); (middle) Comparison of TDOS of freestanding LSMO(001) thin film with LSMO(001) PDOSes of pentacene/LSMO(001) (a) and 2-ZGNR/LSMO(001) of energetically favorable $\eta^{l}$ configurations; (bottom) Comparison of TDOSes of freestanding pentacene (a) and 2-ZGNR (b) with PDOSes of both fragments. For spatial distribution of spin density, blue color corresponds to spin down and green color corresponds to spin up density. TDOSes of pentacene/LSMO(001) (a) and 2-ZGNR/LSMO(001) (b) are presented in black solid lines, TDOSes of free-standing LSMO, pentacene and 2-ZGNR are presented in red solid lines, PDOSes of SMO, pentacene and 2-ZGNR fragments of pentacene/LSMO(001) and 2-ZGNR/LSMO(001) hetersotructures are presented in blue solid lines. 
The electronic density distribution and differential electronic density distribution (both in $e / \AA^{3}$ ) perpendicular to the pentacene/LSMO $(001)$ interface are presented in Figure 5a. The maxima in electronic density distribution correspond to the electronic subsystems localized on pentacene (top) and $\mathrm{SrO}, \mathrm{MnO}$ and $\mathrm{LaO}$ layers of LSMO substrate, respectively. The global minimum in electronic density corresponds to the middle of pentace-LSMO(001) distance. The differential electronic density distribution was calculated as a difference between the spatial distribution of the electronic density of pentacene/LSMO $(001)$ heterostructure and superposition of the electronic densities of free-standing pentacene and LSMO(001) fragments. One can see that formation of the heterostructure causes complex charge redistribution in the interface region with the shift of the electronic density localized on the top of $\mathrm{SrO}$ layer and $\mathrm{MnO}$ layer towards pentacene molecule. The biggest positive amplitude of differential electronic density, attributed to pentacene molecule is located from vacuum side (upper the pentacene plane, Figure 5a, middle). The negative differential amplitude is located right below the pentacene plane, making pentacene polarized in perpendicular direction of pentacene plane. The positive (upper pentacene plane) and negative (below the pentacene plane) parts of differential density almost compensate each other giving small total $0.06 e$ charge transfer to pentacene molecule. The charge transfer from the top LSMO $\mathrm{SrO}$ and $\mathrm{MnO}$ layers to interface region and the spatial shift of the charge closer to the pentacene molecule can be interpreted in terms of structure induced asymmetry and interface 2D electronic gas of pioneering work of Bychkov and Rashba. ${ }^{18}$ In comparison with pentacene, LSMO substrate is a vast reservoir of the electrons and the shift of a small portion of electronic charge from it causes less relative perturbation of the fragment electronic subsystem than the same electronic transfer from pentacene molecule. The charge transfer from LSMO substrate to the interface region (Figure 4a, TDOS and PDOS of LSMO) causes decreasing of the total occupation number of LSMO fragment and small consequent shift of LSMO PDOS towards the Fermi level.

Comparative symmetry analysis of localized molecular orbitals (HOMO, HOMO-1 and LUMO) of free-standing pentacene (Figure 5b, Left) and spin-up and spin-down quasi-molecular pentacene HOMO and LUMO states of pentacene/LSMO(001) heterostructure (Figure 5b, Right) clearly demonstrates that both spin-up and spin-down HOMO and LUMO partners keep intact original molecular symmetry of the parent molecular states. Indirect electronic exchange interactions of spin-down states of LSMO and pentacene electronic subsystems cause deep energy shift of spin-down states, making spin-down quasi-molecular pentacene LUMO state just $0.2 \mathrm{eV}$ higher in energy the spin-up HOMO.

Figure 4a, top, clearly demonstrates distinctive spin-down density, localized at the interface on the top of oxygen ions of $\mathrm{SrO}$ terminal layer of $\operatorname{LSMO}(001)$ surface. Indirect electron exchange interactions of spin-down pentacene states with electron-deficit spin-down states of LSMO, partially localized on $\mathrm{Op}_{z}$ orbitals of oxygen atoms belong to both surface $\mathrm{SrO}$ and inner $\mathrm{MnO}$ layers is the main reason of deep energy shift of spin-down pentacene subsystem.

The qualitative analysis of pentacene PDOS (Figure 5b, Right) reveals small charge transfer from pentacene fragment HOMO (small portion of the orbital is located upper the Fermi 
level) to the interface region (Figures 5a, middle). Complex charge transfer mechanism causes significant PDOS redistribution (new secondary peaks appear in LSMO PDOS, Figure 4a) and red shift of LSMO PDOS.

Freestanding pentacene molecule has closed electronic shell of diamagnetic nature, so its spin-up and spin-down states bear the same spatial distribution (Figure 5b, left). The oxygen ions of $\mathrm{SrO}$ interface layer - which cause induced spin polarization of pentacene molecule through indirect exchange mechanism coupled with structural asymmetry at the interface ${ }^{18}$ - are negatively spin-polarized (Figure 4a, top). Extra spin-down density at the interface region results in increasing of exchange interactions within spin-down subsystem and significant (almost $1 \mathrm{eV}$, Figure 5b, right) blue energy shift of spin-down pentacene quasi-molecular orbitals. The spin-up and spin-down HOMOs keep the same spatial symmetry with very different energy positions of the states caused by different values of Coulomb and exchange interactions among spin-up and spin-down subsystems. Conservation of spatial symmetry of frontier pentacene orbitals directly demonstrate retention of pentacene electronic subsystem in pentacene/LSMO(001) heterostructure.

a)
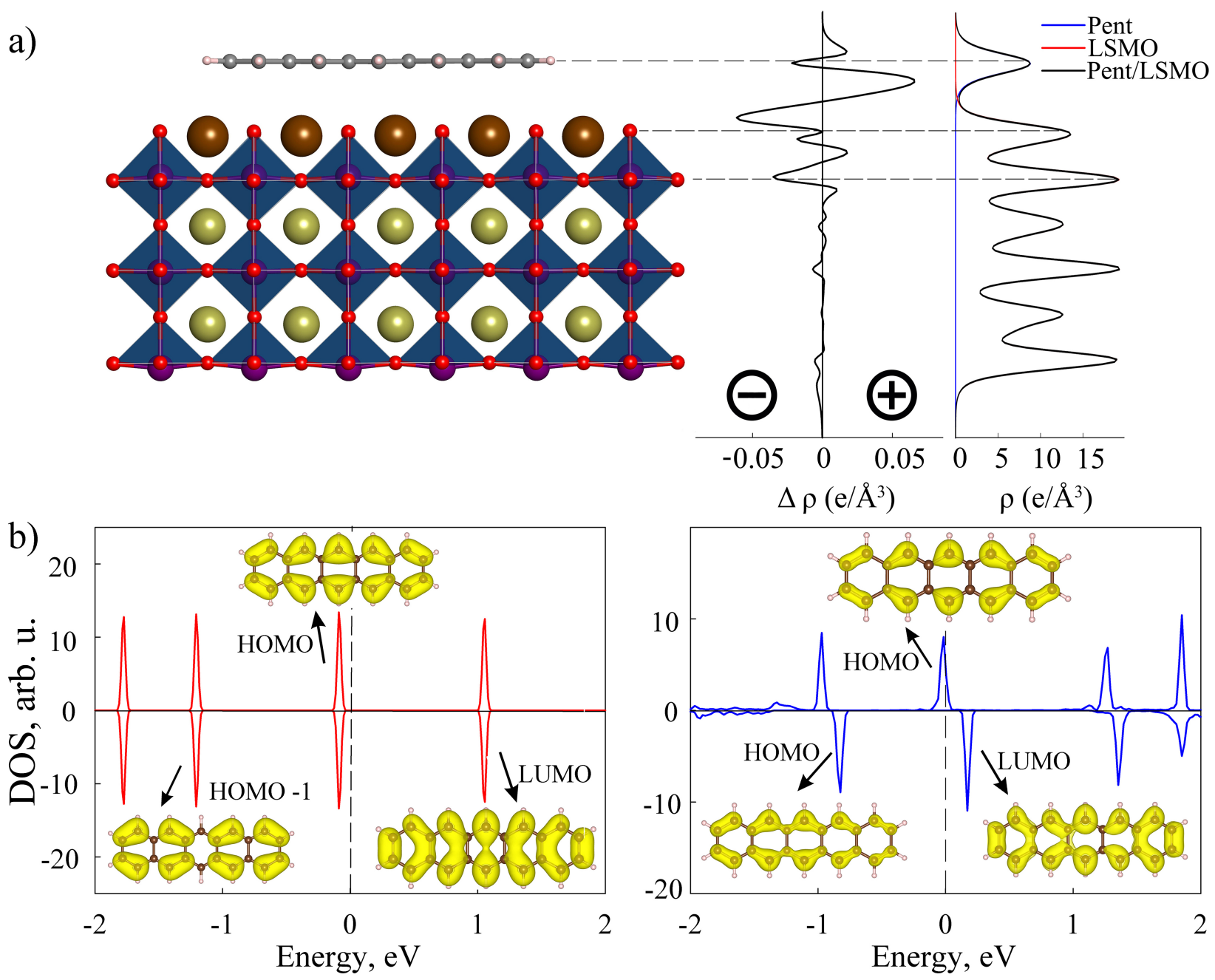
Figure 5. (a) The electronic density $\left(e / \AA^{3}\right)$ distribution (right) and differential electronic density distribution (center) in perpendicular direction of pentacene/LSMO(001) heterostructure (left). The dashed lines, which indicate positions of pentacene, $\mathrm{SrO}$ surface plane and $\mathrm{MnO}$ plane, are the guides for the eyes. The plus and minus signs designate the spatial regions of charge transfer from LSMO(001) and to pentacene fragments. (b) Density of states and spatial distribution of electronic density of pentacene. Left: Freestanding pentacene HOMO (top), HOMO-1 (bottomleft) and LUMO (bottom-right) states. Right: Localized quasi-molecular HOMO spin-up (top), HOMO spin-down (bottom-left) and LUMO spin-down (bottom-right) states of pentacene fragment. The conservation of the symmetries of HOMO and LUMO spin-up and spin-down states is clearly seen for pentacene quasi-molecular states.

The Bader analysis reveals small magnetic moments localized at $\mathrm{Sr}(<0.01 \mu \mathrm{B})$ and oxygen $(0.06 \mu \mathrm{B})$ surface ions. The magnetic moments of pentacene carbon atoms are much higher and equal to $0.01-0.12 \mu \mathrm{B}$. Since surface Sr ions are almost spin neutral, spin-polarized $\mathrm{MnO}_{6}$ octahedrons are the only source of induced spin polarization of carbon atoms caused by indirect exchange mechanism through the surface SrO layer.

The same nature of spin polarization is observed for 2-ZGNR on LSMO(001) (Figure 4b). The TDOS of 2-ZGNR/LSMO(001) heterostructure is determined by LSMO fragment. Spinpolarization caused by structure asymmetry at the interface and indirect exchange through surface oxygen ions converts antiferromagnetic 2-ZGNR with semiconducting band gap of 0.36 $\mathrm{eV}$ (bottom of Figure $4 \mathrm{~b}$ ) into $100 \%$ spin-polarized half-metal with $0.36 \mathrm{eV}$ spin-down band gap. The spin densities of $\mathrm{Sr}$ and $\mathrm{O}$ ions at the $\operatorname{LSMO}(001)$ surface are close to ones for pentacene/LSMO(001) (see above). Spin polarization of 2-ZGNR is much smaller than the pentacene one and equals to $0.01 \mu \mathrm{B}$ on the edge 2-ZGNR carbon atoms and $0.00 \mu \mathrm{B}$ on central ones. 

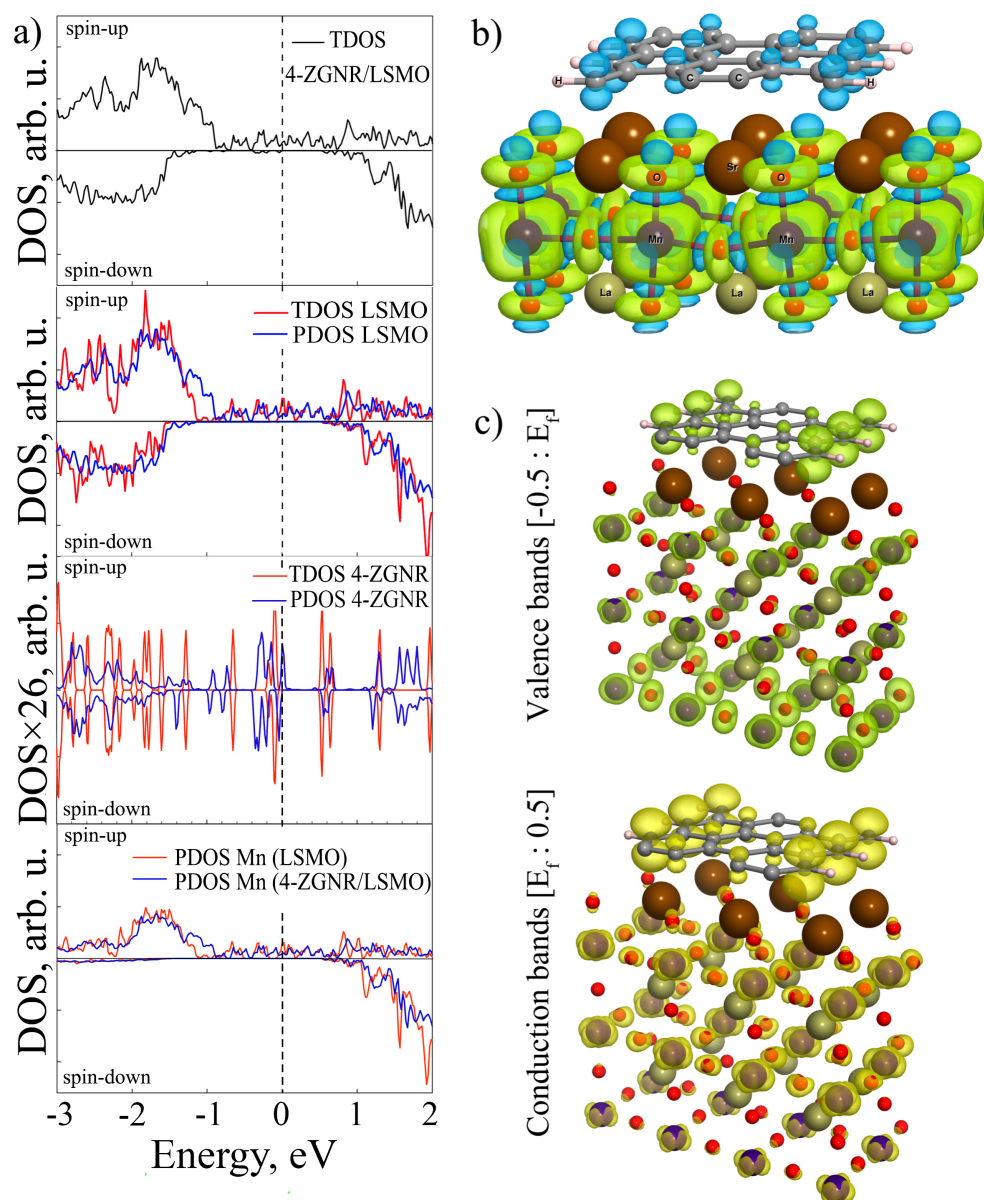

Figure 6. a) Top: Spin-up and spin-down total density of states of 4-ZGNR/LSMO(001) (black solid lines). The next from the top: Partial density of states (blue solid lines) of 4-ZGNR and LSMO(001) fragments. Bottom: PDOS of Mn ions of LSMO fragment. For the sake of comparison, total density of states of free-standing 4-ZGNR and LSMO(001) are presented as well (red solid lines). PDOSes of Mn ions of 4-ZGNR/LSMO(001) heterostructure (blue line) and free-standing LSMO (red ine) are presented as blue and red solid lines, respectively. b) 4ZGNR/LSMO(001) integral spin density spatial distribution in the whole energy region. c) Integral spatial distribution of electron density for the top of valence band (from $-0.5 \mathrm{eV}$ to $\mathrm{E}_{f}$ ) and bottom of conduction band (from $\mathrm{E}_{\mathrm{f}}$ to $0.5 \mathrm{eV}$ ). For the spin density, the blue color corresponds to spin down and the green color corresponds to spin up components, respectively.

The total and partial density of states and spatial distribution of integral spin density of 4ZGNR/LSMO(001) composite are presented in Figures 6. LSMO substrate is responsible for $100 \%$ spin-polarization of the heterostructure TDOS (top of Figure 6a). In contrast with pentacene/LSMO(001) and 2-ZGNR/LSMO(001), LSMO PDOS of 4-ZGNR/LSMO(001) in the region from -1.2 to $1.0 \mathrm{eV}$ is affected by symmetry lowering and indirect electronic exchange interactions with relatively large carbon fragment which cause conversion of semiconducting antiferromagnetic pristine 4-ZGNR (Figure 6a) with $0.62 \mathrm{eV}$ band gap into $100 \%$ spin-polarized half-metal with $0.60 \mathrm{eV}$ spin-down band gap. The splitting energy of spin-up and spin-down 
components is small and equals to $0.04 \mathrm{eV}$, which is less than the accuracy of DFT approach. The very small value of the splitting can vanish as a result of even small structural distortions as well and it can be regarded as negligible.

According to Bader analysis, the total magnetic moment on the edge carbon atoms of 4ZGNR is equal to $0.01 \mu \mathrm{B}$. It is clearly seen (Figure 6b) that integral spin density in the whole energy region is localized mostly at the edge carbon atoms. The distribution of integral electron density of valence and conduction bands in the frontier regions from $-0.5 \mathrm{eV}$ up to $\mathrm{E}_{\mathrm{f}}(0.0 \mathrm{eV})$ and from $E_{\mathrm{f}}$ to $0.5 \mathrm{eV}$ is presented in Figure $6 \mathrm{c}$. The electronic states at the top of the valence band and bottom of conduction band are localized at both LSMO and 4-ZGNR fragments. It is necessary to note that frontier states belong to the edge carbon atoms of 4-ZGNR (Figure 6b), so these atoms would be responsible for transport properties of the composite.

The spin polarization of ZGNRs and pentacene follows LSMO(001) substrate positive spin direction. The preservation of the sign of spin polarization shows that the spin transport in the composites is strong and it could cause large magnetoresistance effect. ${ }^{61}$ This effect is very important to develop organic spin valves.

The structure of 4-ZBNNR/LSMO(001) interface is much more complex due to the presence of two types of atoms (nitrogen and boron) in the unit cell of zigzag $h$-BN nanoribbon (Figure 7). In contrast with carbon-based heterostructures, all 4-ZBNNR/LSMO(001) configurations reveal noticeably shorter interlayer distances in the range of $2.761-2.821 \AA$. Six configurations of 4-ZBNNR/LSMO were located by electronic structure calculations (Table 3 ) with the lowest in energy $\eta^{1-2-3-6}$ one (Figure 7e). All configurations have almost identical binding energies which are weaker than the binding energy of the most stable 4ZGNR/LSMO(001) $\eta^{3}$ configuration $\left(-0.210 \mathrm{eV}\right.$ per $\mathrm{C}_{2}$ dimer), which is typical energy of vdW bonding (see above). 
a)

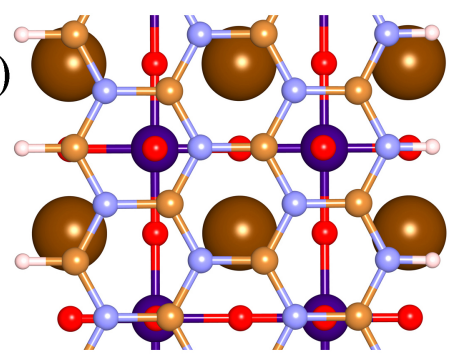

c)

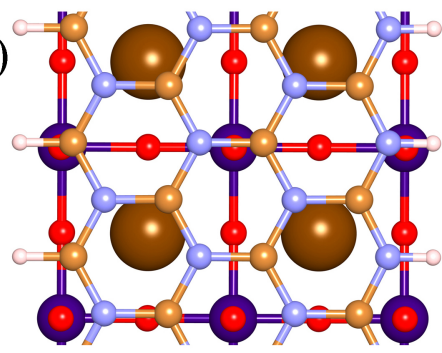

e)

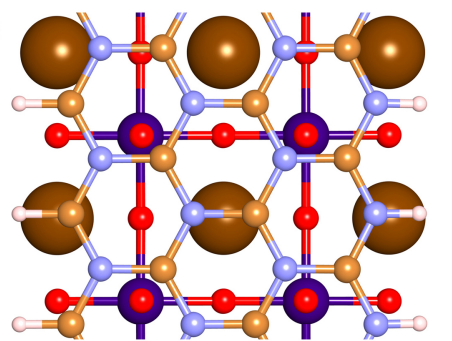

- $-\mathrm{Sr} \odot-\mathrm{Mn}$

- N b)

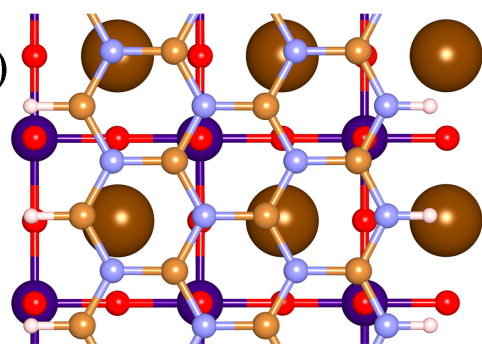

d)

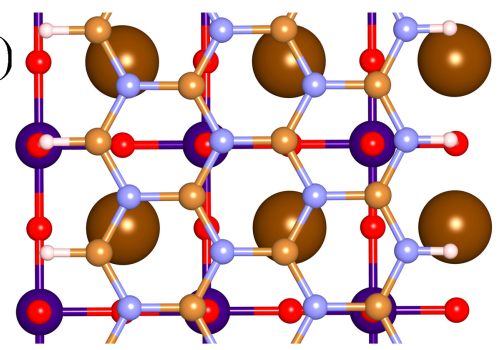

f)

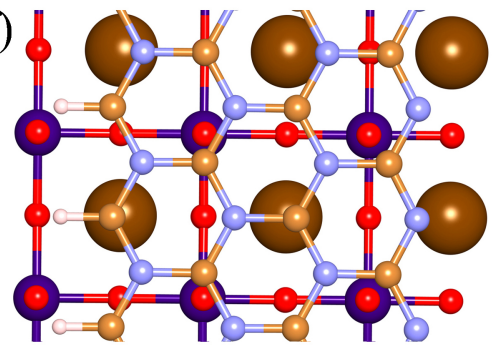

$-\mathrm{B}$ g)
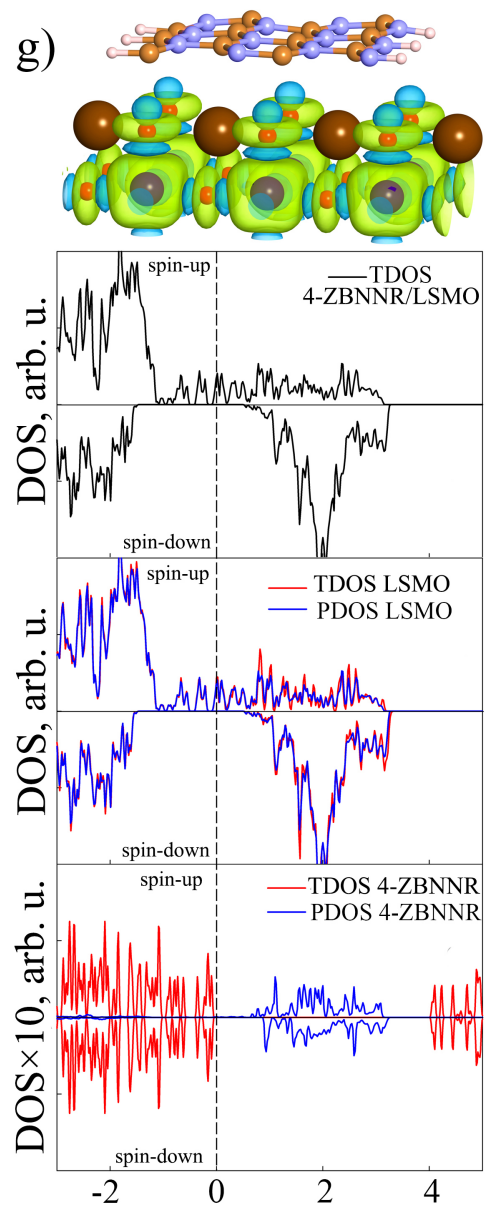

Energy, eV

Figure 7. Located configurations of 4-ZBNNR/LSMO heterostructure. a) $\eta^{l-2}$ configuration; b) $\eta^{l}$ configuration; c) $\eta^{3}$ configuration; d) $\eta^{2}$ configuration; e) energetically favorable $\eta^{1-2-3-6}$ configuration; f) $\eta^{1-3}$ configuration. g) Spatial distribution of spin density (top) and spin-up and spin-down density of states (second, third and forth panels) of 4-ZBNNR/LSMO heterostructure, namely: 4-ZBNNR/LSMO TDOS (black solid lines); TDOS of free-standing LSMO (red solid line) and PDOS of LSMO fragment in 4-ZBNNR/LSMO heterostructure (blue solid line); TDOS of free-standing 4-ZBNNR (red solid line) and PDOS of 4-ZBNNR fragment in 4ZBNNR/LSMO(001) heterostructure (blue solid line). All DOSes are calculated for energetically preferable 4-ZBNNR/LSMO(001) $\eta^{1-2-3-6}$ configuration.

Table 3. Binding energies and average interlayer distances of 4-ZBNNR/LSMO(001) heterostructure.

\begin{tabular}{ccccccc}
\hline & \multicolumn{5}{c}{ 4-ZBNNR/LSMO(001) } \\
\cline { 2 - 7 } Configuration & $\eta^{1-2}$ & $\eta^{l}$ & $\eta^{3}$ & $\eta^{2}$ & $\eta^{1-2-3-6}$ & $\eta^{1-3}$ \\
\hline $\begin{array}{c}\text { Binding energy, eV } \\
\text { (Binding energy }\end{array}$ & -1.670 & -1.556 & -1.568 & -1.552 & -1.677 & -1.621 \\
per BN pair, eV) & $(-0.139)$ & $(-0.130)$ & $(-0.131)$ & $(-0.129)$ & $(-0.140)$ & $(-0.135)$ \\
\hline
\end{tabular}




\begin{tabular}{ccccccc}
\hline $\begin{array}{c}\text { Average interlayer } \\
\text { distance, } \AA\end{array}$ & 2.821 & 2.821 & 2.761 & 2.769 & 2.777 & 2.782 \\
\hline
\end{tabular}

Freestanding 4-ZBNNR is characterized by wide band gap (4.04 eV, Figure $7 \mathrm{~g}$ bottom) which prevents an appearance of half-metallic electronic configuration of 4-ZBNNR fragment. The formation of the 4-ZBNNR/LSMO $(001)$ heterostructure leads to formation of embedded electronic states inside $h$-BN band gap with spin-up and spin-down fragment gaps of 0.70 and $0.98 \mathrm{eV}$, respectively. The spin polarization of 4-ZBNNR caused by $h-\mathrm{BN}$ fragment spin-up and spin-down asymmetrical embedded states could be interpreted as a combined effect of interface structural asymmetry and indirect exchange interactions of the heterostructure fragments.

\section{Conclusions}

Using DFT $+U$ calculations it was shown that promising low-dimensional LSMO(001)based heterostructures composed by combination of a number of infinite and finite graphene (pentacene molecule, 2-ZGNR and 4-ZGNR) and $h$-BN (4-ZBNNR) zigzag nanoribbons reveal several competing interfacial configurations formed by weak dispersion interactions between the fragments. Vertical structural asymmetry of pentacene/LSMO(001) heterostructure and indirect exchange interactions of surface oxygen ions of $\mathrm{MnO}_{6}$ octahedrons of LSMO substrate with pentacene molecule lead to deep shift of pentacene localized quasi-molecular spin-down electronic states and strong spin polarization of the molecule. The interfragment interactions convert antiferromagnetic 2-ZGNR and 4-ZGNR fragments to $100 \%$ spin-polarized half-metals with $3.1-3.2 \AA$ interlayer distances. The preservation of the positive sign of spin polarization shows that the spin transport in the composites is strong, which could cause large magnetoresistance effect. Structural asymmetry and indirect exchange interactions with LSMO support also cause spin polarization of 4-ZBNNR fragment with creation of embedded states in the forbidden band gap in 4-ZBNNR PDOS. The electronic structure calculations clearly demonstrate a possibility of development of promising LSMO-based low-dimensional heterostructures with zigzag graphene and $h$-BN nanoribbons for spin-related applications.

\section{Acknowledgements}

We acknowledge the Siberian Supercomputer Center (SSCC) of SB RAS, Novosibirsk; the Joint Supercomputer Center of RAS, Moscow and the ICC of Novosibirsk State University for providing the computing resources. P.B.S. acknowledges the financial support of the Ministry of Education and Science of the Russian Federation in the framework of Increase Competitiveness Program of NUST «MISiS» (№ K2-2015-033) Russian Science Foundation (Grant No 14-13-00139) supported the work of the Russian team. 


\section{References}

(1) Marrows, C. H.; Hickey, B. J. New directions in spintronics. Philos. Trans. A. 2011, 369, 3027-3036.

(2) Naber, W. J. M.; Faez, S.; van der Wiel, W. G. Organic spintronics. J. Phys. D. Appl. Phys., 2007, 40, R205-R228.

(3) Shiraishi, M.; Ikoma, T. Molecular spintronics. Phys. E Low-dimensional Syst. Nanostructures, 2011, 43, 1295-1317.

(4) Tombros, N.; Jozsa, C.; Popinciuc, M.; Jonkman, H. T.; van Wees, B. J. Electronic spin transport and spin precession in single graphene layers at room temperature. Nature, 2007, 448, 571-574.

(5) Kuzubov, A.A.; Kovaleva, E.A.; Avramov, P.; Kuklin, A.V.; Mikhaleva, N.S.; Tomilin, F.N.; Sakai, S.; Entani, S.; Matsumoto Y.; Naramoto, H. Contact-induced spin polarization in $\mathrm{BNNT}(\mathrm{CNT}) / \mathrm{TM}(\mathrm{TM}=\mathrm{Co}, \mathrm{Ni})$ nanocomposites. J. Appl. Phys., 2014, 116, 084309.

(6) Tsukagoshi K.; Alphenaar, B.W.; Ago, H. Coherent transport of electron spin in a ferromagnetically contacted carbon nanotube. Nature, 1999, 401, 572-574.

(7) Xiong, Z.H.; Wu, D.; Vardeny Z.V.; Shi, J. Giant magnetoresistance in organic spinvalves. Nature, 2004, 427, 821-824.

(8) Petta, J.R.; Slater S.K.; Ralph, D.C. Spin-Dependent Transport in Molecular Tunnel Junctions. Phys. Rev. Lett., 2004, 93, 136601.

(9) Sakai, S.; Yakushiji, K.; Mitani, S.; Takanashi, K.; Naramoto, H.; Avramov, P.V.; Narumi, K.; Lavrentiev V.; Maeda, Y. Tunnel magnetoresistance in Co nanoparticle/Co-C 60 compound hybrid system. Appl. Phys. Lett., 2006, 89, 113118.

(10) Sakai, S.; Sugai, I.; Mitani, S.; Takanashi, K.; Matsumoto, Y.; Naramoto, H.; Avramov, P.V.; Okayasu, S.; Maeda, Y. Giant tunnel magnetoresistance in co-deposited fullerenecobalt films in the low bias-voltage regime. Appl. Phys. Lett., 2007, 91, 242104.

(11) Xue, J.; Sanchez-Yamagishi, J.; Bulmash, D.; Jacquod, P.; Deshpande, A.; Watanabe, K.; Taniguchi, T.; Jarillo-Herrero, P.; LeRoy, B.J. Scanning tunnelling microscopy and spectroscopy of ultra-flat graphene on hexagonal boron nitride. Nat. Mater., 2011, 10, 282-285.

(12) Kamalakar, M.V.; Dankert, A.; Bergsten, J.; Ive, T.; Dash, S.P. Enhanced tunnel spin injection into graphene using chemical vapor deposited hexagonal boron nitride. Sci. Rep., 2014, 4, 6146.

(13) Kamalakar, M.V.; Dankert, A.; Kelly, P.J.; Dash, S.P. Inversion of Spin Signal and Spin Filtering in Ferromagnet|Hexagonal Boron Nitride-Graphene van der Waals Heterostructures. Sci. Rep., 2016, 6, 21168.

(14) Huang, B.; Monsma, D.J.; Appelbaum, I. Coherent Spin Transport through a 350 Micron Thick Silicon Wafer. Phys. Rev. Lett., 2007, 99, 177209.

(15) Huang, B.; Appelbaum, I. Spin dephasing in drift-dominated semiconductor spintronics devices. Phys. Rev. B, 2008, 77, 165331.

(16) Rashba, E.I. Theory of electrical spin injection: Tunnel contacts as a solution of the conductivity mismatch problem. Phys. Rev. B - Condens. Matter Mater. Phys., 2000, 62, R16267-R16270.

(17) Varykhalov, A.; Sánchez-Barriga, J., Shikin, A.M.; Biswas, C.; Vescovo, E.; Rybkin, A.; Marchenko, D.; Rader, O. Electronic and Magnetic Properties of Quasifreestanding Graphene on Ni. Phys. Rev. Lett., 2008, 101, 157601. 
(18) Bychkov, Y.A.; Rashba, E.I. Properties of a 2D electron gas with lifted spectral degeneracy. Sov. Phys. JETP Lett., 1984, 39, 78-81.

(19) Zútic, I.; Fabian, J.; Das Sarma, S. Spintronics: Fundamentals and applications. Rev. Mod Phys., 2004, 76, 323-410.

(20) Krupin, O.; Bihlmayer, G.; Starke, K.; Gorovikov, S.; Prieto, J.E.; Dóbrich, K.; Blügel, S.; Kaindl, G. Rashba effect at magnetic metal surfaces. Phys. Rev. B, 2005, 71, 201403(R).

(21) Nagaev, E.L. Lanthanum manganites and other giant-magnetoresistance magnetic conductors. Physics-Uspekhi, 1996, 39, 781-805.

(22) Perkalina, T.M.; Kotjuzhansky, B.Ya.; Shapiro, A.Ya.; Cherkezyan, S.A. Magnetic and elastic properties of La0.9Sr0.1Mn1-xGaxO3+ gamma-compounds. Fiz. Tverd. Tela (in Russian) 1990, 32, 1242-1245.

(23) Ruderman, M.A.; Kittel, C. Indirect Exchange Coupling of Nuclear Magnetic Moments by Conduction Electrons. Phys. Rev., 1954, 96, 99-102.

(24) Kasuya, T. Electrical resistance of ferromagnetic metals. Prog. Theor. Phys., 1956, 16, 45-57.

(25) Yosida, K. Magnetic Properties of Cu-Mn Alloys. Phys. Rev., 1957, 106, 893-898.

(26) Nagaev, E.L. Phase-separation mechanism for giant magnetoresistance of lanthanum manganites. Phys. Lett. A, 1996, $218,367-372$.

(27) Tamura, S. Magnetic measurements of $(\mathrm{La} 0.8 \mathrm{Ca} 0.2) \mathrm{MnO} 3+\mathrm{y}$ by the Faraday method. Phys. Lett. A, 1980, 78, 401-403.

(28) Yokota, T.; Murata, S.; Gomi, M. Electric field-induced magnetic changes in $\mathrm{La} 0.7 \mathrm{Sr} 0.3 \mathrm{MnO} 3$ thin film using electric field-induced resistance phenomenon. Appl. Phys. Lett., 2013, 102, 152404.

(29) de Groot, R.A.; Mueller, F.M.; van Engen, P.G.; Buschow, K.H.J. New Class of Materials: Half-Metallic Ferromagnets. Phys. Rev. Lett., 1983, 50, 2024-2027.

(30) Jin, S.; Tiefel, T.H.; McCormack, M.; Fastnacht, R.A.; Ramesh, R.; Chen, L.H. Thousandfold Change in Resistivity in Magnetoresistive La-Ca-Mn-O Films. Science 1994, 264, 413-415.

(31) Park, J.-H.; Vescovo, E.; Kim, H.-J.; Kwon, C.; Ramesh, R.; Venkatesan, T. Direct evidence for a half-metallic ferromagnet. Nature 1998, 392, 794-796.

(32) Viret, M.; Nassar, J.; Drouet, M.; Contour, J.; Fermon, C.; Fert, A. Spin polarised tunnelling as a probe of half metallic ferromagnetism in mixed-valence manganites. $J$. Magn. Magn. Mater., 1999, 198-199, 1-5.

(33) Ziese, M. Extrinsic magnetotransport phenomena in ferromagnetic oxides. Reports Prog. Phys., 2002, 65, 143-249.

(34) De Teresa, J.M.; Barthelemy, A.; Fert, A.; Contour, J.P.; Montaigne, F.; Seneor, P. Role of Metal-Oxide Interface in Determining the Spin Polarization of Magnetic Tunnel Junctions. Science, 1999, 286, 507-509.

(35) Davis, A.H.; Bussmann, K. Organic luminescent devices and magnetoelectronics. J. Appl. Phys., 2003, 93, 7358.

(36) Wang, F.J.; Yang, C.G.; Vardeny, Z.V.; Li, X.G. Spin response in organic spin valves based on La2/3Sr1/3MnO3 electrodes. Phys. Rev. B, 2007, 75, 245324.

(37) Rocci, M.; Tornos, J.; Rivera-Calzada, A.; Sefrioui, Z.; Clement, M.; Iborra, E.; Leon, C.; Santamaria, J. Resistive switching in manganite/graphene hybrid planar nanostructures. Appl. Phys. Lett., 2014, 104, 102408.

(38) Li, F.; Li, T.; Guo, X. Vertical graphene spin valves based on La2/3Sr1/3MnO3 electrodes. 
ACS Appl. Mater. Interfaces, 2014, 6, 1187-1192.

(39) Wang, X.; Wang, P.; Wang, J.; Hu, W.; Zhou, X.; Guo, N.; Huang, H.; Sun, S.; Shen, H.; Lin, T.; Tang, M.; Liao, L.; Jiang, A.; Sun, J.; Meng, X.; Chen, X.; Lu, W.; Chu, J. Ultrasensitive and broadband MoS2 photodetector driven by ferroelectrics. Advanced Materials, 2015, 27, 6575-6581.

(40) Sakai, S.; Majumdar, S.; Popov, Z.I.; Avramov, P.V.; Entani, S.; Hasegawa, Y.; Yamada, Y.; Huhtinen, H.; Naramoto, H.; Sorokin, P.B.; Yamauchi, Y. Proximity-Induced Spin Polarization of Graphene in Contact with Half-Metallic Manganite. ACS Nano, 2016, 10, 7532-7541. DOI: 10.1021/acsnano.6b02424

(41) Hueso, L.E.; Pruneda, J.M.; Ferrari, V.; Burnell, G.; Valdés-Herrera, J.P.; Simons, B.D.; Littlewood, P.B.; Artacho, E.; Fert, A.; Mathur, N.D. Transformation of spin information into large electrical signals using carbon nanotubes. Nature, 2007, 445, 410-413.

(42) Graziosi, P.; Riminucci, A.; Prezioso, M.; Newby, C.; Brunel, D.; Bergenti, I.; Pullini, D.; Busquets-Mataix, D.; Ghidini, M.; Dediu, V.A. Pentacene thin films on ferromagnetic oxide: Growth mechanism and spintronic devices. Appl. Phys. Lett., 2014, 105, 022401.

(43) Chu, Y.-H.; Hsu, C.-H.; Lu, C.-I.; Yang, H.-H.; Yang, T.-H.; Luo, C.-H.; Yang, K.-J.; Hsu, S.-H.; Hoffmann, G.; Kaun, C.-C.; Lin, M.-T. Spin-Dependent Molecule Symmetry at a Pentacene-Co Spinterface. ACS Nano, 2015, 9, 7027-7032.

(44) Son, Y.-W.; Cohen, M.L.; Louie, S.G. Energy Gaps in Graphene Nanoribbons. Phys. Rev. Lett., 2007, 97, 216803.

(45) Li, Y.; Cohen, M.L.; Louie, S.G. Magnetic Edge-State Excitons in Zigzag Graphene Nanoribbons. Phys. Rev. Lett., 2008, 101, 186401.

(46) Baringhaus, J.; Ruan, M.; Edler, F.; Tejeda, A.; Sicot, M.; Taleb-Ibrahimi, A.; Li, A.-P.; Jiang, Z.; Conrad, E.H.; Berger, C.; Tegenkamp, C.; de Heer, W.A. Exceptional ballistic transport in epitaxial graphene nanoribbons. Nature, 2014, 506, 349-354.

(47) Castro Neto, A.H.; Peres, N.M.R.; Novoselov, K.S.; Geim, A.K. The electronic properties of graphene. Rev. Mod. Phys., 2009, 81, 109-162.

(48) Perdew, J.P.; Burke, K.; Ernzerhof, M. Generalized Gradient Approximation Made Simple. Phys. Rev. Lett., 1996, 77, 3865-3868.

(49) Kresse, G.; Hafner, J. Ab initio molecular-dynamics simulation of the liquid-metalamorphous-semiconductor transition in germanium. Phys. Rev. B, 1994, 49, 14251-14269.

(50) Kresse, G.; Furthmüller, J. Efficient iterative schemes for ab initio total-energy calculations using a plane-wave basis set. Phys. Rev. B, 1996, 54, 11169-11186.

(51) Anisimov, V.I.; Zaanen, J.; Andersen, O.K. Band theory and Mott insulators: Hubbard U instead of Stoner I. Phys. Rev. B, 1991, 44, 943-954.

(52) Dudarev, S.L.; Savrasov, S.Y.; Humphreys, C.J.; Sutton, A.P. Electron-energy-loss spectra and the structural stability of nickel oxide: An LSDA+U study. Phys. Rev. B, 1998, 57, 1505-1509.

(53) Grimme, S. Semiempirical GGA - type density functional constructed with a long - range dispersion correction. J. Comput. Chem., 2006, 27, 1787-1799.

(54) Kresse, G. From ultrasoft pseudopotentials to the projector augmented-wave method. Phys. Rev. B, 1999, 59, 1758-1775.

(55) Blöchl, P.E. Projector augmented-wave method. Phys. Rev. B, 1994, 50, 17953-17979.

(56) Ma, C.; Yang, Z.; Picozzi, S. Ab initio electronic and magnetic structure in La0. 66Sr0. 33MnO3: strain and correlation effects. J. Phys. Condens. Matter, 2006, 18, 7717-7728.

(57) Picozzi, S.; Ma, C.; Yang, Z.; Bertacco, R.; Cantoni, M.; Cattoni, A.; Petti, D. ; Brivio, S.; 
Ciccacci, F. Oxygen vacancies and induced changes in the electronic and magnetic structures of La0.66Sr0.33MnO3: A combined ab initio and photoemission study. Phys. Rev. B, 2007, 75, 94418 .

(58) Zheng, B.; Binggeli, N. Influence of the interface atomic structure on the magnetic and electronic properties of $\mathrm{La} 2 / 3 \mathrm{Sr} 1 / 3 \mathrm{MnO} 3 / \mathrm{SrTiO} 3(001)$ heterojunctions. Phys. Rev. B, 2010, 82, 245311.

(59) Monkhorst, H.J.; Pack, J.D. Special points for Brillouin-zone integrations. Phys. Rev. B, 1976, 13, 5188-5192.

(60) Tsui, F.; Smoak, M.C.; Nath, T.K.; Eom, C.B. Strain-dependent magnetic phase diagram of epitaxial La0.67Sr0.33MnO3 thin films. Appl. Phys. Lett., 2000, 76, 2421.

(61) Gross, L.; Mohn, F.; Moll, N.; Liljeroth, P.; Meyer, G. The Chemical Structure of a Molecule Resolved by Atomic Force Microscopy. Science, 2009, 325, 1110-1114.

(62) Henkelman, G.; Arnaldsson, A.; Jónsson, H. A fast and robust algorithm for Bader decomposition of charge density. Comput. Mater. Sci., 2006, 36, 354-360.

(63) Sanville, E.; Kenny, S.D.; Smith, R.; Henkelman, G. Improved grid - based algorithm for Bader charge allocation. J. Comput. Chem., 2007, 28, 899-908.

(64) Tang, W.; Sanville, E.; Henkelman, G. A grid-based Bader analysis algorithm without lattice bias. J. Phys. Condens. Matter, 2009, 21, 084204.

(65) Atkins, P.W.; De Paula, J. Physical chemistry for the life sciences, W.H. Freeman and Co., 2011.

(66) Pruneda, J.M.; Ferrari, V.; Rurali, R.; Littlewood, P.B.; Spaldin, N.A.; Artacho, E. Ferrodistortive Instability at the (001) Surface of Half-Metallic Manganites. Phys. Rev. Lett., 2007, 99, 226101.

(67) Maurice, J.-L.; Imhoff, D.; Contour, J.-P.; Colliex, C. Interfaces in $\{100\}$ epitaxial heterostructures of perovskite oxides. Philos. Mag., 2006, 86, 2127-2146.

(68) Petrov, A.Y.; Torrelles, X.; Verna, A.; Xu, H.; Cossaro, A.; Pedio, M.; Garcia-Barriocanal, J.; Castro, G.R.; Davidson, B.A. Surface octahedral distortions and atomic design of perovskite interfaces. Adv. Mater., 2013, 25, 4043-4048.

(69) Poggini, L.; Ninova, S.; Graziosi, P.; Mannini, M.; Lanzilotto, V.; Cortigiani, B.; Malavolti, L.; Borgatti, F.; Bardi, U.; Totti, F.; Bergenti, I.; Dediu, V.A.; Sessoli, R. A combined ion scattering, photoemission, and DFT investigation on the termination layer of a La0.7Sr0.3Mno3 spin injecting electrode. J. Phys. Chem. C, 2014, 118, 13631-13637.

(70) Yu, P.; Luo, W.; Yi, D.; Zhang, J.X.; Rossell, M.D.; Yang, C.-H.; You, L.; Singh-Bhalla, G.; Yang, S.Y.; He, Q.; Ramasse, Q.M.; Erni, R.; Martin, L.W.; Chu, Y.H.; Pantelides, S.T.; Pennycook, S.J.; Ramesh, R. Interface control of bulk ferroelectric polarization. Proc. Natl. Acad. Sci., 2012, 109, 9710-9715.

(71) Yoshimoto, M.; Maruta, H.; Ohnishi, T.; Sasaki, K.; Koinuma, H. In situ determination of the terminating layer of $\mathrm{La} 0.7 \mathrm{Sr} 0.3 \mathrm{MnO} 3$ thin films using coaxial impact-collision ion scattering spectroscopy. Appl. Phys. Lett., 1998, 73, 187-189.

(72) Boschker, H.; Verbeeck, J.; Egoavil, R.; Bals, S.; van Tendeloo, G.; Huijben, M.; Houwman, E.P.; Koster, G.; Blank, D.H.A.; Rijnders, G. Preventing the reconstruction of the polar discontinuity at oxide heterointerfaces. Adv. Funct. Mater., 2012, 22, 2235-2240.

(73) Liao, Z.; Huijben, M.; Zhong, Z.; Gauquelin, N.; Macke, S.; Green, R.J.; van Aert, S.; Verbeeck, J.; van Tendeloo, G.; Held, K.; Sawatzky, G.A.; Koster, G.; Rijnders, G. Controlled lateral anisotropy in correlated manganite heterostructures by interfaceengineered oxygen octahedral coupling. Nat. Mater., 2016, 15, 425-431. 
(74) Zhang, Q.; Yin, L.; Mi, W.; Wang, X. Large Spatial Spin Polarization at Benzene/La2/3Sr1/3MnO3 Spinterface: Toward Organic Spintronic Devices. J. Phys. Chem. C, 2016, 120, 6156-6164. 